\title{
Repeated in vivo Visualization of Neuromuscular Junctions in Adult Mouse Lateral Gastrocnemius
}

\author{
Donald J. Wigston \\ Department of Physiology, Emory University School of Medicine, Atlanta, Georgia 30322
}

The structure of individually identified neuromuscular junctions (NMJs) in mouse lateral gastrocnemius (LG) muscles was studied on 2 or more occasions over 3-6 months. Presynaptic motor nerve terminals and their underlying acetylcholine receptors were stained in living animals with the fluorescent dye 4-(4-diethylaminostyryl)- $\mathbf{N}$-methylpyridinium iodide) and tetramethylrhodamine isothiocyanate-conjugated $\alpha$-bungarotoxin ( $R \alpha B T X)$, respectively, and visualized by video-enhanced fluorescence microscopy. The overall shape of most NMJs changed very little over this time except for enlargement of some junctions attributable to growth of the animals. A few junctions did, however, change appreciably: over 3-6 months about $15 \%$ underwent modifications such as additions to, or losses from, their original configuration. The frequency and extent of changes in LG NMJs were substantially less than in a similar study of NMJs from mouse soleus (Wigston, 1989). These findings, together with those from other laboratories, indicate a correlation between the extent of NMJ remodeling and the fiber-type composition of a muscle: NMJs in muscles consisting of predominantly fast-twitch myofibers appear to undergo less remodeling than NMJs in muscles containing a substantial fraction of slow-twitch fibers. Since fast- and slow-twitch muscles and their motoneurons exhibit strikingly different patterns of electrical activity, these findings suggest that structural remodeling at mammalian NMJs may depend on the amount of impulse activity experienced by motoneurons, their target muscle, or individual synaptic terminals.

One view of how the nervous system changes with experience is that individual synaptic connections are dynamic and respond to altered input or activation with a change in their performance. Although much work is currently aimed at clucidating the biochemical and pharmacological mechanisms of synaptic plasticity (Castellucci et al., 1986; Teyler and DiScenna, 1987; Nicoll et al., 1988; Ito, 1989; Zucker, 1989), it is likely that short-term changes in synaptic efficacy are stabilized by morphological alterations at individual synapses (Bailey and Chen, 1988a, b; Grenough and Bailey, 1988). Using video-enhanced low-lightlevel fluorescence microscopy it has recently become feasible to

\footnotetext{
Received Oct. 25, 1989; revised Dec. 13, 1989; accepted Dec. 15, 1989.

I would like to thank J. Huffaker, A. Shirley, and S. Mayer for assistance, and R. Balice-Gordon, V. Boss, A. English, J. Lichtman, and D. Purves for helpful suggestions and advice. This work was supported by grants from MDA and NIH.

Correspondence should be addressed to Dr. Donald J. Wigston at the above address.

Copyright (C) 1990 Society for Neuroscience $0270-6474 / 90 / 061753-09 \$ 02.00 / 0$
}

observe identified synaptic connections at multiple times in living animals (Purves and Voyvodic, 1987). In this way, it is possible to determine directly whether the structure or location of individual synapses is static, or whether it is continually changing. For instance, it has been shown that presynaptic boutons on mammalian autonomic ganglion cells undergo dramatic remodeling during normal adult life (Purves et al., 1987).

Other investigators have exploited the vertebrate neuromuscular junction (NMJ) to examine the question of synaptic remodeling (Lichtman et al., 1987; Robbins and Polak, 1987; Wigston, 1989; Herrera et al., 1990): the large size and accessibility of the NMJ and the availability of several fluorescent probes that reveal its pre- and postsynaptic structure are advantages for in vivo imaging of nerve-muscle synapses. The case for ongoing remodeling at the frog NMJ is quite clear. Both traditional, histological approaches (Wernig et al., 1980) and modern in vivo imaging methods (Herrera et al., 1990) have demonstrated that frog NMJs typically undergo substantial modifications to their shape over several months. In contrast to the frog NMJ, the extent of synaptic remodeling at the mammalian NMJ is not so clear. In spite of histological indications that mammalian NMJs were likely to be continuously remodeled (Cardasis and Padykula, 1981; Wernig et al., 1984), one recent study of mouse sternomastoid using in vivo imaging gave little indication of synaptic rearrangement over a 6-month period (Lichtman et al., 1987). However, NMJs in a different muscle, mouse soleus, undergo significant remodeling (Wigston, 1989). Indeed, much of the prior histological evidence that had indicated the likelihood that mammalian NMJs underwent remodeling was obtained in soleus.

The apparent difference in the frequency of remodeling observed in sternomastoid and soleus might be explained if NMJs in some muscles underwent substantial remodcling, whereas NMJs in other muscles did not. Since mouse soleus contains a large proportion of slow-twitch, or tonic, muscle fibers and sternomastoid contains predominantly fast-twitch, or phasic, fibers, it is conceivable that NMJs on slow-twitch fibers might exhibit more structural plasticity than NMJs on fast-twitch fibers, perhaps related to the differences in their electrical or contractile activity. Another possibility is that remodeling of NMJs actually does occur in sternomastoid, but is not well revealed by 4-(4diethylaminostyryl)- $N$-methylpyridinium iodide (4-Di-2-ASP), the only probe employed by Lichtman et al. (1987), which may not completely delineate all parts of every presynaptic nerve terminal, especially in older animals (Balice-Gordon and Lichtman, 1990). Indeed, some of the synaptic remodeling observed by Wigston (1989) in mouse soleus was postsynaptic and was not accompanied by noticeable changes in the shape of the 
Figure 1. Diagram of the method used to obtain images of NMJs in LG of living mice. $A$, LG was exposed by making an incision in the overlying skin. The skin and the biceps femoris muscle which lies over LG were then retracted, revealing the anterior surface of LG, which is normally apposed to the posterior surface of soleus. $B, A$ 12-mmdiameter coverglass was applied to the surface of LG with a micromanipulator, the mouse and its supporting platform were placed on the stage of a compound microscope, and the muscle was positioned under a high numerical aperture water-immersion objective. The muscle and coverglass were bathed in oxygenated mammalian Ringer's throughout the procedure.
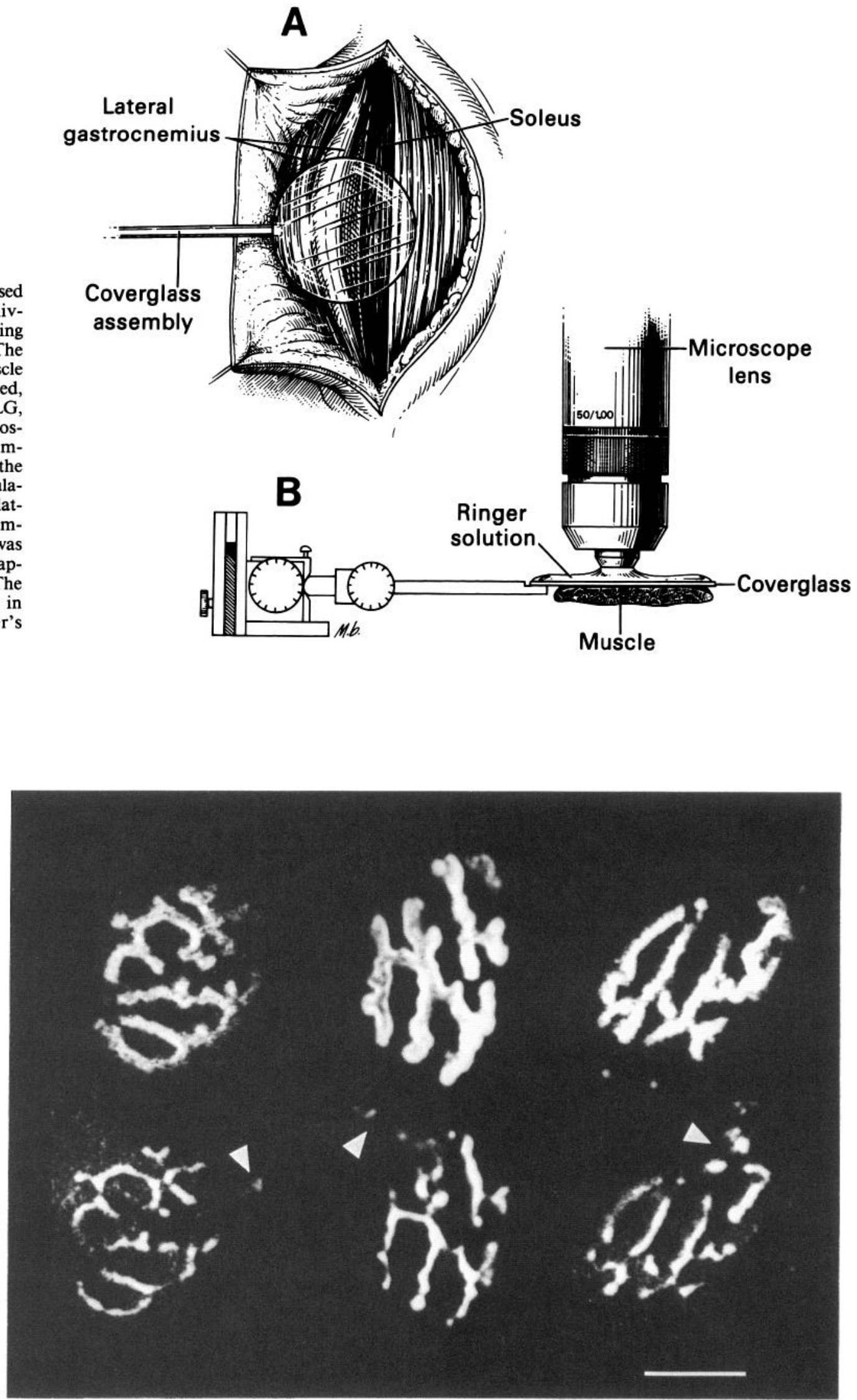

Figure 2. Correspondence of 4-Di-2-ASP and R- $\alpha$ BTX staining of NMJs. Three different NMJs are shown stained with R- $\alpha$ BTX (top panels) to delineate the arrangement of acetylcholine receptors, and 4-Di-2-ASP (bottom) to reveal nerve terminals. Note the alignment of nerve terminals and receptors. Receptors, however, are not associated with the myelinated, preterminal axon (arrowheads). Scale bar, $25 \mu \mathrm{m}$. 


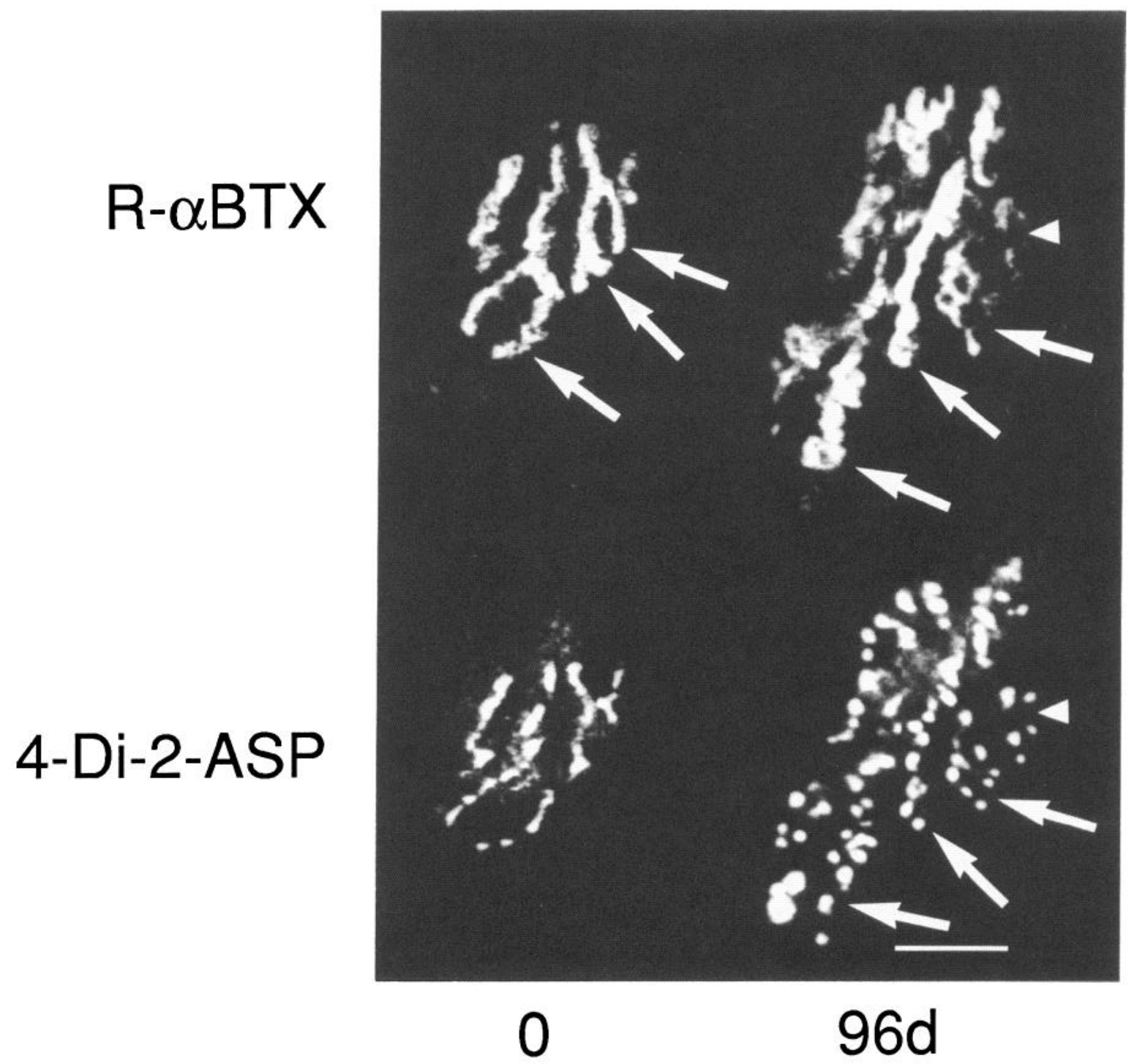

Figure 3. Example of NMJ examined on 2 occasions, with 3 months intervening. This junction was visualized with R- $\alpha \mathrm{BTX}$ (upper panels) and 4-Di-2-ASP (lower panels) initially (left) and again $96 \mathrm{~d}$ later (right). The overall size of the junction increased substantially (approximately $2.7 \times$ increase in area), and the 4-Di-2-ASP staining took on a spotty appearance. Common reference points are marked with large arrows. Furthermore, a new branch $15 \mu \mathrm{m}$ in length was apparent by the second viewing at the far right of the junction (arrowheads in right panels). It is unlikely that this branch was simply obscured at time 0 since the staining with both R- $\alpha$ BTX and 4-Di-2-ASP was quite good at this first viewing. Furthermore, NMJs stained with R- $\alpha$ BTX could be seen clearly on the deep surface of other muscle fibers in this and other muscles studied. Scale bar, $25 \mu \mathrm{m}$.

presynaptic terminal: regions of receptor staining disappeared from underneath short segments of nerve terminal, close to where the axonal myelin sheath ended. This form of NMJ remodeling would not have been detected using only a nerve terminal probe.

For these reasons I now report the results of a study of 99 NMJs in mouse lateral gastrocnemius (LG), a predominantly fast-twitch hindlimb muscle adjacent to soleus. This study employed both 4-Di-2-ASP and tetramethylrhodamine isothiocyanate-conjugated $\alpha$-bungarotoxin (R $\alpha \mathrm{BTX}$ ) to reveal pre- and postsynaptic specializations of NMJs in LG muscles of living mice. My findings indicate that although some LG NMJs do undergo structural remodeling over a 3- or 6-month period, fewer LG NMJs show anatomical changes than in soleus over comparable periods. Some of these results have been reported previously (Wigston, 1988).

\section{Materials and Methods}

Preparation of animals. Young adult mice of either sex (C57BL/6J strain; weight 22-30 gm, 3-6 months of age) were anesthetized with ketamine and xylazine (100 mg ketamine: $20 \mathrm{mg}$ xylazine per $\mathrm{kg}$ I.P.). A polyethylene tube was then inserted through the mouth into the trachea. The lateral surface of the right hindlimb was shaved and washed with antiseptic solution. The LG muscle was revealed by incising the overlying skin and detaching the biceps femoris muscle at its anterior margin from the tibia. The anterior surface of LG adjacent to soleus was exposed by retracting the overlying fascia in a posterior direction and R $\alpha$ BTX $(\sim 5 \mu \mathrm{g} / \mathrm{ml}$ in sterile oxygenated Ringer's; Molecular Probes) was applied for 5-10 min. This dose resulted in adequate staining of acetylcholine receptors at NMJs but did not block neuromuscular transmission (Wigston, 1989). Unbound toxin was then removed and the muscle rinsed several times with oxygenated Ringer's. A coverslip was then gently applied to the surface of the muscle using a micromanipulator (Fig. 1), the animal was placed on the stage of an epifluorescence microscope, and the endotracheal tube was connected to a respirator. 


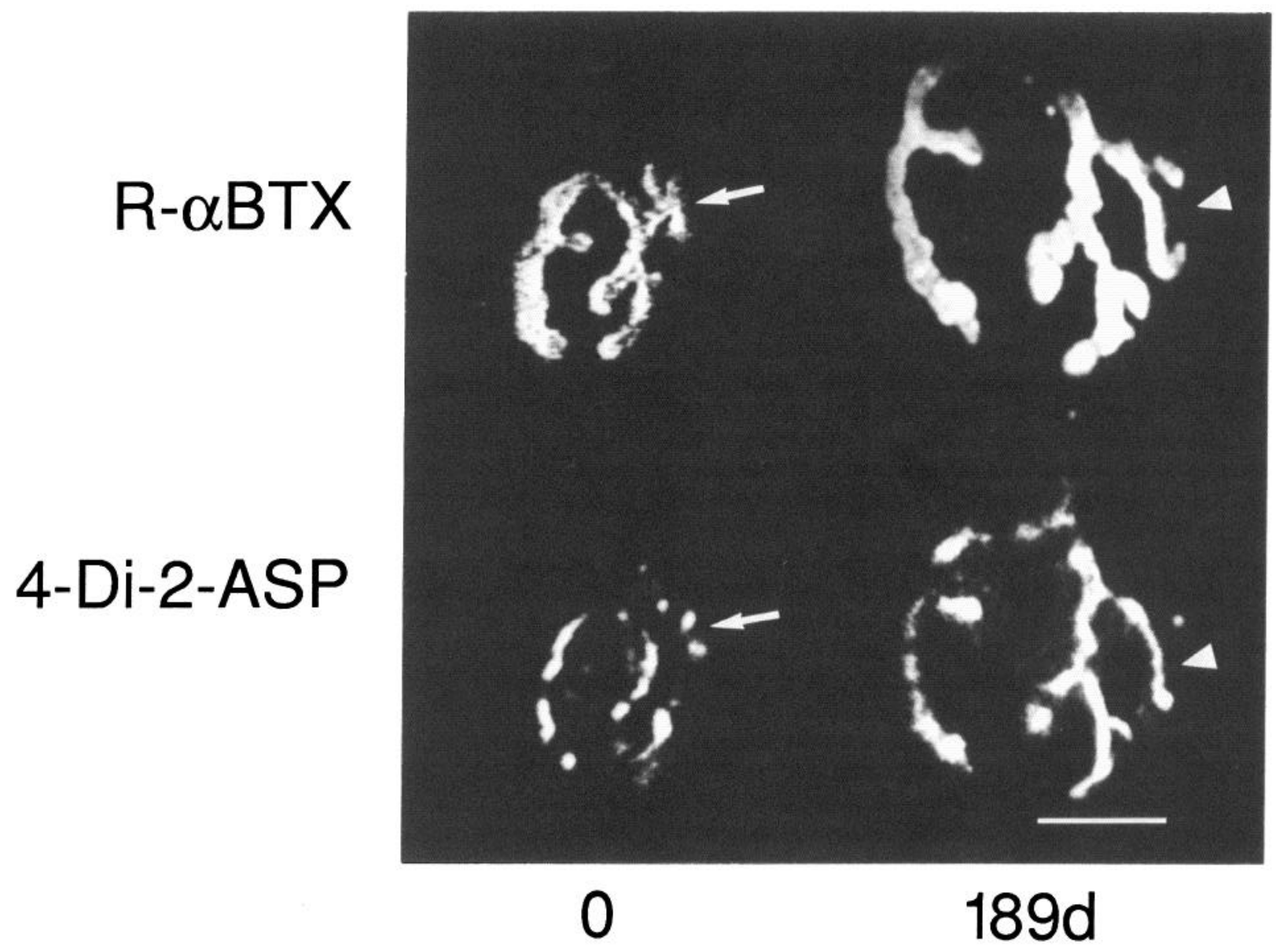

Figure 4. Example of an NMJ studied 6 months after a prior viewing. This NMJ also grew $(2.2 \times$ increase in area) between the first and the second viewing $189 \mathrm{~d}$ later. The branch at the upper right at day 0 (arrows) changed its shape and lengthened considerably over this time (arrowheads in right panels). Scale bar, $25 \mu \mathrm{m}$.

Acetylcholine receptor imaging. NMJs on superficial myofibers in LG labeled with $\mathrm{R} \alpha \mathrm{BTX}$ were then visualized as described by Wigston (1989). Briefly, NMJs were illuminated with a $100 \mathrm{~W}$ mercury arc lamp attenuated with heat filters and a graded neutral density filter, using the Leitz N2 fluorescence filter set (530-560 nm excitation), and viewed with a water-immersion objective lens (Leitz 50X, NA $=1.0$ ). The microscope image was projected to a SIT camera (Dage 66) operated

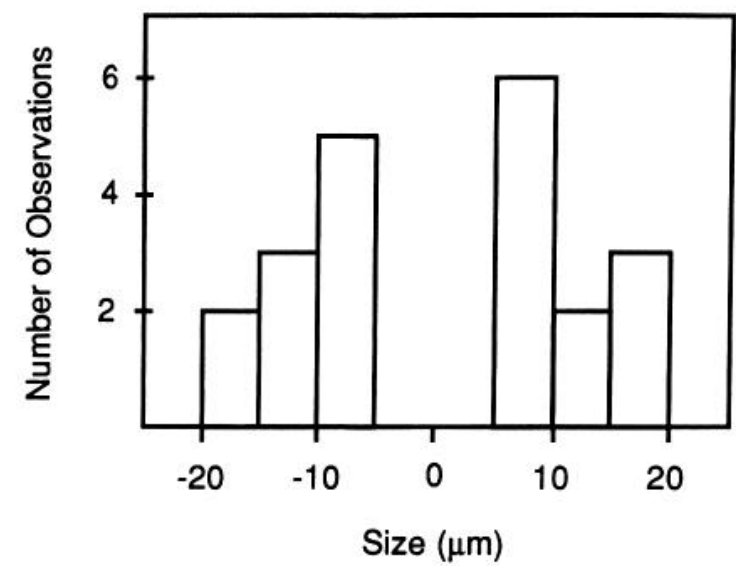

Figure 5. Size distribution of changes in NMJ morphology. Each measurable change exceeding $5 \mu \mathrm{m}$ (additions are positive, deletions negative) is represented. About equal numbers of additions and deletions were observed. at maximum gain, directly coupled to the microscope. No trinocular head or eyepieces were attached to the microscope. This enabled the illumination to be kept low; less than $5 \%$ of the lamp output was required to obtain adequate video images. The signal from the SIT camera was led to a video-rate digital image processor (Image-1 AT, Universal Imaging Corporation). Continuously digitized live images were recorded on videotape using a VCR (Panasonic AG 1950) to aid in subsequent relocation of NMJs. Images of selected junctions were processed to reduce noise ( 32 frame average), background images were subtracted, and contrast was enhanced by expanding the range of gray values. Processed images were recorded on magnetic disks and later transferred to optical disks using a WORM drive (Maxtor).

Table 1. Frequency of remodeling at NMJs in mouse gastrocnemius: comparison of NMJs studied twice with those studied 3 times

\begin{tabular}{lll} 
& 2 views & 3 views \\
\hline Number of animals & 12 & 7 \\
Number of NMJs & 63 & 36 \\
Percent of NMJs changed $^{a}$ & 17 & 8 \\
Changes per NMJ $^{b}$ & $0.29 \pm 0.1$ & $0.12 \pm 0.08$
\end{tabular}

${ }^{a}$ Regions of an NMJ that appeared de novo, grew disproportionately to the rest of the synapse, or disappeared completely were measured. Only individual changes of $5 \mu \mathrm{m}$ or greater were included. Some NMJs exhibited multiple changes.

${ }^{b}$ Mean \pm SE. Calculated by dividing the number of changes observed in a particular muscle by the number of NMJs studied in that muscle and then averaging these values for all muscles within each group. Difference between groups was not significantly different $(p>0.2$; Student's $t$ test). 


\section{R- $\alpha \mathrm{BTX}$}
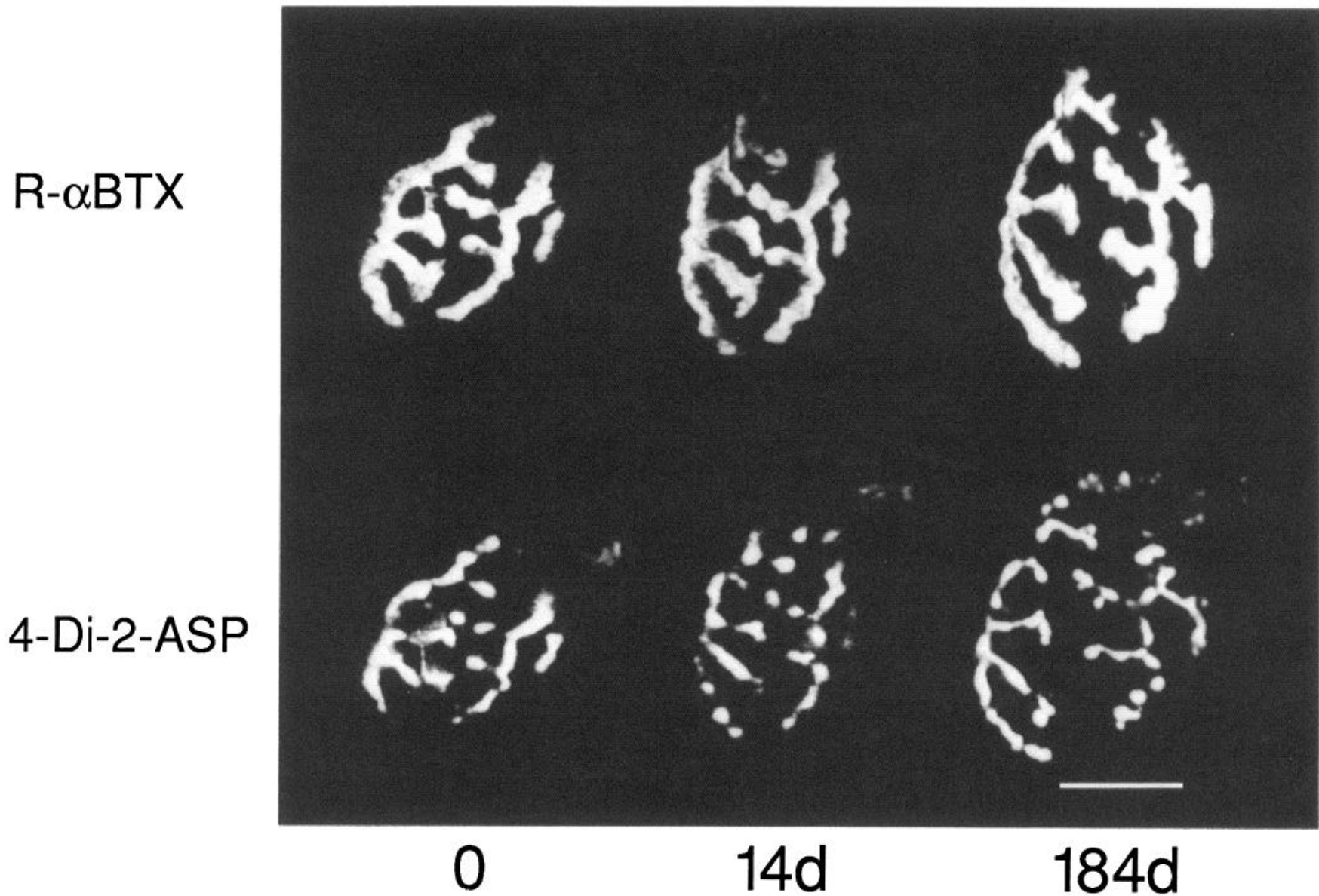

Figure 6. NMJ studied on 3 occasions over 6 months. Except for some overall growth, this NMJ appeared identical at the initial viewing (left), intermediate viewing (center), and final viewing (right) $184 \mathrm{~d}$ after the first. Scale bar, $25 \mu \mathrm{m}$.

Nerve terminal imaging. Since 4-Di-2-ASP-stained nerve terminals could be seen even when the rhodamine (N2) filter set was in position, nerve terminal imaging had to be done after receptor images were obtained. After R $\alpha$ BTX images were studied at 4-9 clearly visible NMJs in each muscle, the coverslip was raised and the muscle incubated for 2-3 min in a $5 \mu \mathrm{M}$ solution of 4-Di-2-ASP (Molecular Probes, Eugene, OR; Magrassi et al., 1987) to stain nerve terminals. The dye was then rinsed out and the coverslip lowered. The motor nerve terminals at the NMJs studied with $\mathrm{R} \alpha \mathrm{BTX}$ were then viewed using a Leitz K2 filter set (470-490 nm excitation), but the camera gain was lowered to improve resolution of fine processes. The greater brightness of the 4-Di2-ASP fluorescence permitted adequate images to be obtained under these conditions of decreased sensitivity without any increase in the intensity of illumination over that used for imaging receptors. The images of nerve terminals could be aligned almost exactly with $\mathrm{R} \alpha \mathrm{BTX}$ images of the same NMJ (Fig. 2). Regions of nonalignment often occurred just beyond the myelin sheath (arrowheads in Fig. 2). Since 4-Di-2-ASP also stains muscle fibers lightly, and stains damaged fibers intensely, any damaged fibers could easily be detected. Muscles that contained damaged fibers, presumably as a result of their surgical exposure, were not studied further. The nerve to LG is not exposed during any part of this procedure and therefore was unlikely to sustain any direct damage. Images of 4-Di-2-ASP fluorescence were processed and stored as above. Animals were then removed from the microscope, their incisions were closed with 7-0 sutures, and they were returned to their cages. At subsequent viewing sessions, the procedure described above was repeated, except that at the final viewing twice the normal concentration of $\mathrm{R} \alpha \mathrm{BTX}$ was applied for a longer time to improve the quality of staining. The NMJs studied previously were relocated using the videotapes of the original viewing session, digital images of the selected NMJs, and their location within the muscle and relationship to one another.
Analysis. Multipanel composite images of each NMJ were constructed so that initial views, intermediate views, and final views could be easily compared. This was done for both nerve terminal and receptor staining images. When changes were apparent, they were measured directly from the video data using image analysis software. For example, if an NMJ lost a branch or part of a branch from its initial structure, the length of the region present initially, but subsequently absent, was measured. Only changes equaling or exceeding $5 \mu \mathrm{m}$ were analyzed. Changes such as the overall enlargement of NMJs, which occurred frequently owing to the growth of the animals over the 6-month maximum observation period, and distortion produced by differential stretching at each viewing were ignored for the purpose of this study. Illustrations of representative NMJs were obtained by photographing composite images produced by the image processor directly from the screen of a video monitor (Sony PVM 122) using a 35-mm camera with automatic exposure control and Kodak Technical Pan film. For NMJs that could not be imaged completely at a single focal plane, in-focus regions of images obtained at different focal planes were combined to form a single image using the image processor.

\section{Results}

\section{Changes in NMJ morphology over 3 months}

The structure of 33 lateral gastrocnemius NMJs in 7 mice was examined with both R $\alpha$ BTX and 4-Di-2-ASP in vivo at 3 months (91-99 d) after an initial observation. Of these, $28(85 \%)$ appeared virtually identical at the initial and final viewings. The remaining $5(15 \%)$, however, exhibited noticeable changes in their shape consisting primarily of additions or deletions from their original structure of up to $20 \mu \mathrm{m}$ (Fig. 3). Of these, some 


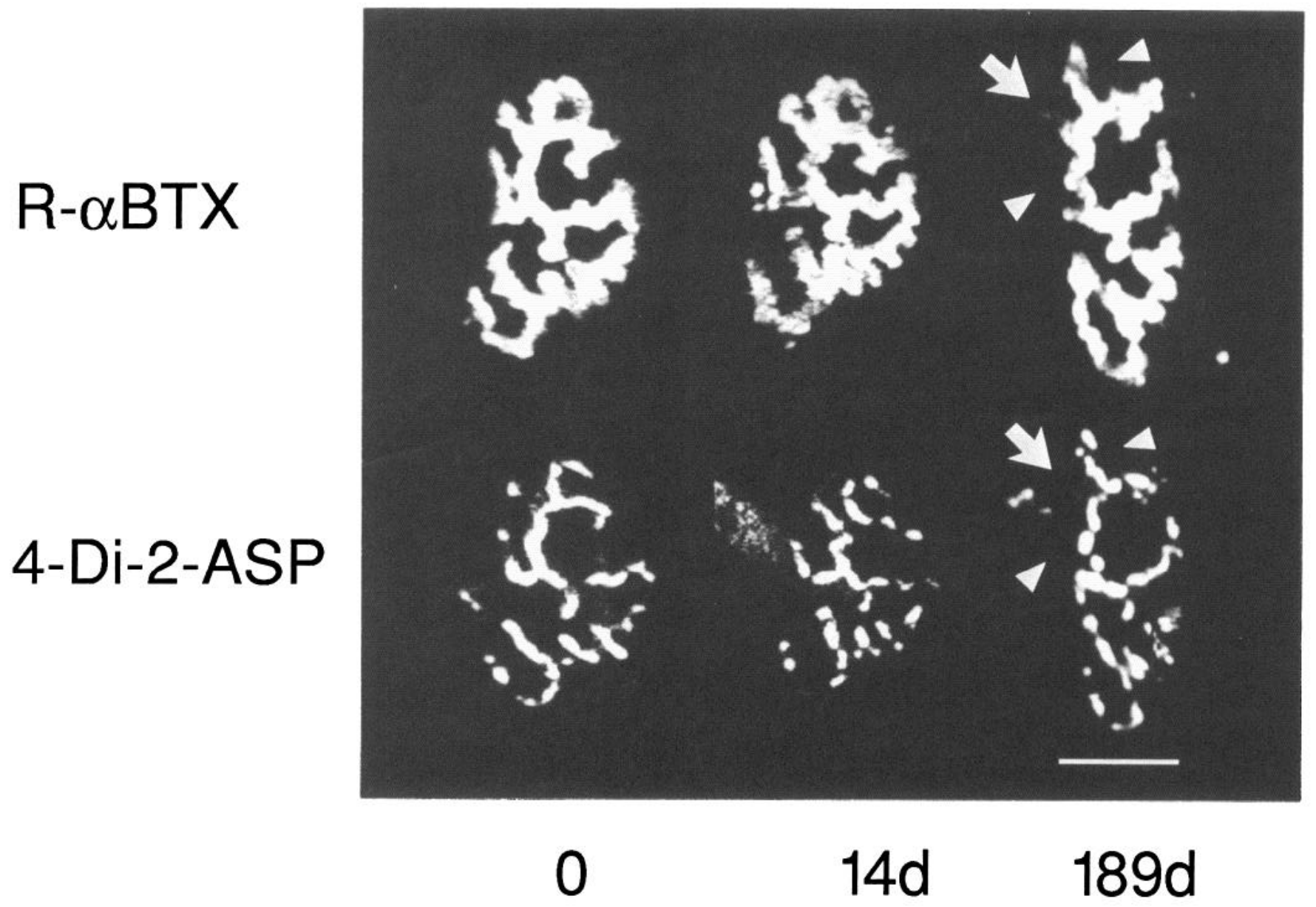

Figure 7. Another NMJ studied on 3 occasions over 6 months. This NMJ underwent minor modifications over the 189-d observation period. At $14 \mathrm{~d}$ (center panels) after the initial observation (left) no changes in either R- $\alpha \mathrm{BTX}$ or 4-Di-2-ASP staining were evident. However, by $189 \mathrm{~d}($ right panels) 2 branches disappeared (arrowheads indicate vacated regions). In addition, the region marked by the large arrow (upper right panel) now is stained only lightly with R- $\alpha \mathrm{BTX}$ (barely visible), indicating that a low density of acetylcholine receptors was still present in this region which had apparently lost its nerve terminal branch (large arrow in bottom right panel). Scale bar, $25 \mu \mathrm{m}$.

$(2 / 5)$ showed multiple changes; for example, one NMJ showed an addition of $16 \mu \mathrm{m}$, as well as a separate deletion of $12 \mu \mathrm{m}$. Changes of less than $5 \mu \mathrm{m}$, or those that could be attributed to differential distortion of NMJ shape at different viewings or the overall growth of an NMJ between viewings, were ignored. Thus, a small fraction of NMJs in lateral gastrocnemius undergo some degree of anatomical remodeling over a period of 3 months.

\section{Changes in NMJ morphology over 6 months}

In another group of 12 mice, for which 6 months (184-193 d) elapsed between the initial and final viewings, 9 of 66 NMJs $(14 \%)$ underwent clear changes in their morphology (e.g., Fig. 4), while the remainder appeared virtually unchanged. Of those that did undergo some modification to their initial structure, $5 / 9$ exhibited 2 or 3 such changes. Two junctions exhibited both deletions and additions. Overall, 8 of 99 junctions studied over 3 or 6 months exhibited deletions, while, similarly, 8 of 99 underwent additions. Thus, noticeable differences between NMJs in LG and soleus were, first, that in LG fewer NMJs exhibited morphological remodeling when studied over 6 months (14\%) than in soleus (44\%; Wigston, 1989); and second, that in LG about equal proportions of deletions and additions occurred
(Fig. 5), whereas in soleus, deletions outnumbered additions considerably (Wigston, 1989).

\section{Comparison of NMJs studied 2 or 3 times in vivo}

To help determine whether there was any effect of the imaging procedure on synaptic morphology, in 7 of the 19 animals described above, NMJs were subjected to an intermediate viewing at 14 days after the initial examination as well as the final viewing at 3 or 6 months. The purpose of this was 2 -fold: first, to determine whether any rearrangement of NMJ morphology was apparent within 2 weeks, which, considering the small number of changes seen after 3 or even 6 months had elapsed, would likely be secondary to damage, and second, to compare the frequency and extent of remodeling at NMJs studied on 2 occasions prior to their final viewing with those studied only once before. If NMJ staining and viewing triggered morphological changes directly, then more changes should be observed in NMJs studied 3 times overall than in those studied only twice. At 14 d, no NMJs exhibited clear changes that satisfied the $\pm 5 \mu \mathrm{m}$ criterion. Many junctions showed no changes even over 6 months (Fig. 6). However several junctions did change (Fig. 7). The frequency of NMJ remodeling, that is, the proportion of NMJs 


\section{4-Di-2-ASP}

\section{$\mathrm{R}-\alpha \mathrm{BTX}$}

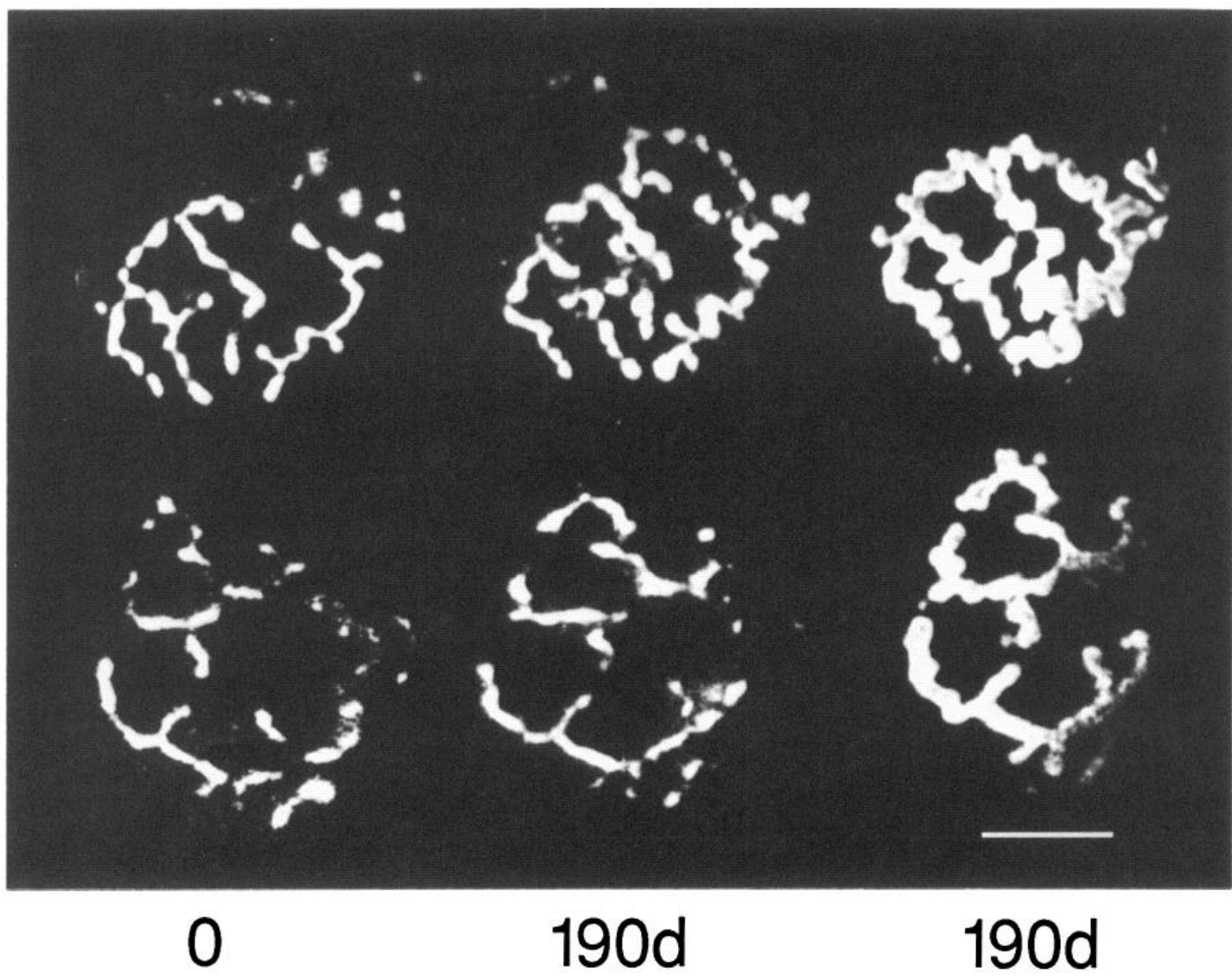

Figure 8. NMJs studied twice over 6 months. These 2 NMJs (first, upper panels; second, lower panels) were visualized initially with 4-Di-2-ASP only (left) and then again $190 \mathrm{~d}$ later with both 4-Di-2-ASP (middle) and R- $\alpha$ BTX (right). The faint staining with 4-Di-2-ASP at the top of the upper NMJ at both time 0 and $190 \mathrm{~d}$ delineates the myelinated segment of the preterminal axon and has no associated R- $\alpha$ BTX staining. Although in the upper NMJ the completeness and intensity of 4-Di-2-ASP staining in the top part of the junction was better at $190 \mathrm{~d}$ than at time 0 , no true remodeling was evident in this NMJ or the one below. Scale bar, $25 \mu \mathrm{m}$.

that exhibited remodeling, was $17 \%$ for those studied on a total of 2 occasions and $8 \%$ for those studied 3 times (Table 1). Thus, under the conditions employed in this study, the staining and viewing of NMJs in vivo did not appear to increase the occurrence of morphological remodeling.

\section{NMJs studied initially with 4-Di-2-ASP only}

One difference between this study, in which NMJ remodeling was observed, and another which showed little if any remodeling to occur (Lichtman et al., 1987) was that in the present study postsynaptic specializations were also stained in vivo with $\mathrm{R} \alpha \mathrm{BTX}$, whereas Lichtman et al. (1987) stained only presynaptic terminals with 4-Di-2-ASP. To further examine the possibility that NMJ remodeling was induced by $\mathrm{R} \alpha \mathrm{BTX}$, even though applied in nonblocking doses (Wigston, 1989; see also Rochel and Robbins, 1987; Rich and Lichtman, 1989a), in 4 of the 12 animals studied over 6 months (described above) 23 NMJs were imaged at first with only 4-Di-2-ASP, and then again 6 months later with both 4-Di-2-ASP and R $\alpha$ BTX. Although most of these NMJs showed no remodeling (Fig. 8), 5 NMJs $(22 \%)$ exhibited changes similar in nature to those seen in NMJs studied with both probes (Fig. 9). Thus, synaptic remodeling occurs whether or not R $\alpha \mathrm{BTX}$ is used initially, demonstrating further that junctional remodeling is not a side effect of $\alpha$-bungarotoxin treatment, but is likely to be part of a normal process of morphological adjustments that occurs during the life of adult mice.

\section{Discussion}

The observations reported here show that the vast majority of NMJs in adult mouse LG are morphologically stable for periods of up to 6 months. Over 6 months, $14 \%$ of the NMJs studied showed minor changes in their structure: some lost branches or parts of branches, while others acquired additional features or grew disproportionately in particular regions. Yet others exhibited changes that were difficult to quantify in this simple manner (for example, Fig. 9). Many NMJs expanded overall during this time, most likely attributable to the growth of the animals and enlargement of their muscle fibers. Thus, in mouse LG, as in soleus, NMJs can undergo changes in their structure, even in adults.

The difference between my present findings in LG and my 


\section{4-Di-2-ASP}

\section{$\mathrm{R}-\alpha \mathrm{BTX}$}

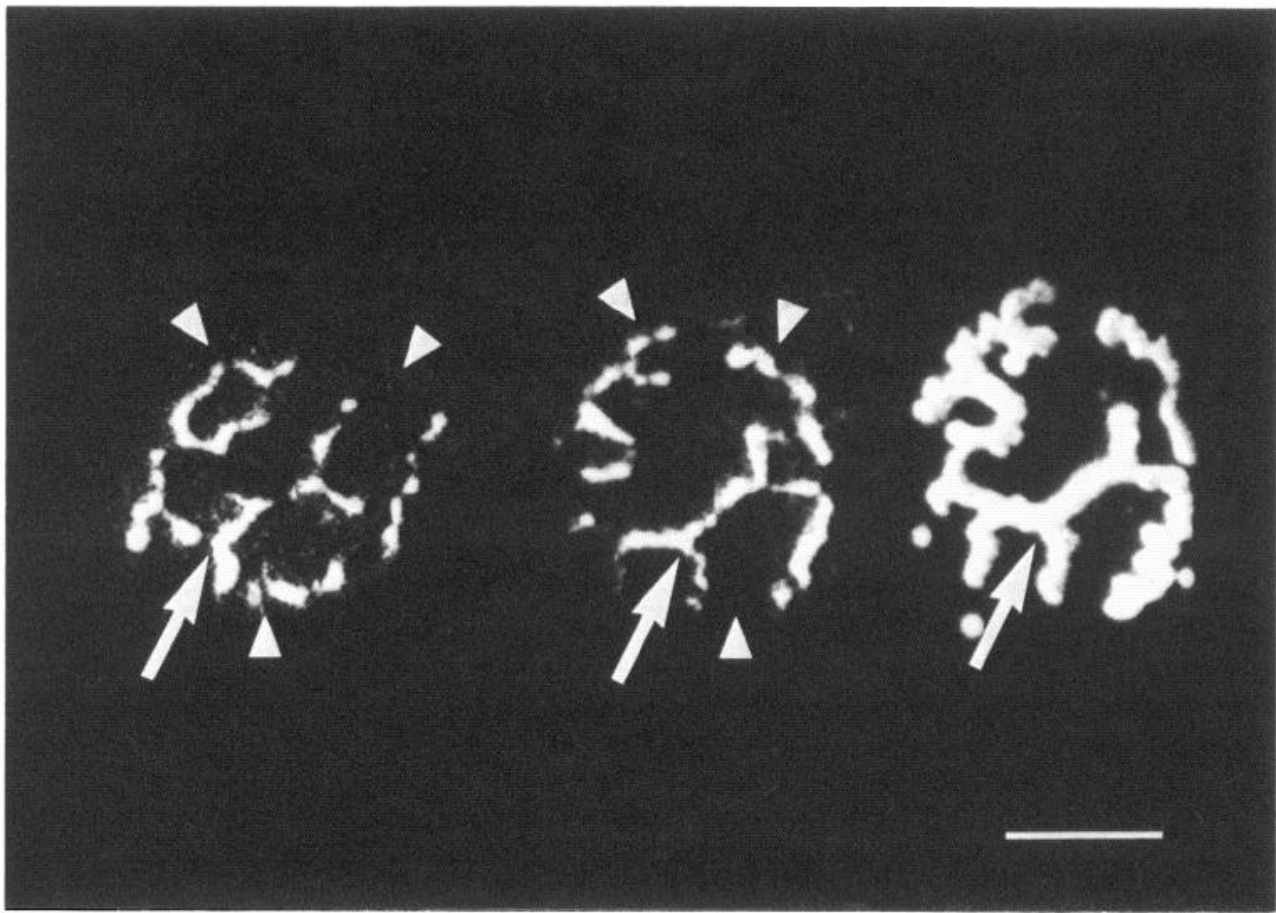

Figure 9. NMJ studied twice over 6 months. This NMJ was studied with 4-Di-2-ASP initially $(l e f t)$ and then again $190 \mathrm{~d}$ later with both 4-Di-2-ASP and R- $\alpha$ BTX (middle and right). A reference point common to all 3 panels is indicated with a large arrow. Note changes in the configuration of the regions indicated by arrowheads in left and middle panels. Scale bar, $25 \mu \mathrm{m}$.
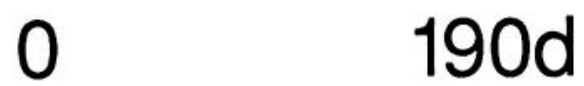

$190 d$

previous findings in soleus may help explain the basis of NMJ remodeling. Using similar methods and conditions to those employed in my study of soleus (Wigston, 1989), I observed far less remodeling of NMJs in LG than in soleus, suggesting a difference between NMJs in LG and soleus. One possible explanation for this difference is based on observations that NMJs in predominantly slow-twitch muscles tend to be relatively small and simple whereas NMJs in predominantly fast-twitch muscles are larger and more complex (Fahim et al., 1984; Lømo and Waerhaug, 1985). Thus remodeling of soleus NMJs may occur secondarily to a conversion of motor units from one fiber type to another. NMJs on myofibers that switched from a fast to a slow type might therefore be remodeled from large, complex shapes typical of fast-twitch fibers to smaller, simpler configurations found in slow-twitch fibers. Such a conversion of motor units from fast-twitch to slow-twitch characteristics has indeed been reported to occur in rat soleus between the ages of 5 weeks and 34 weeks (Kugelberg, 1976), perhaps in response to the increased load placed on the muscle during growth of the animal. Although I used animals at least 3 months old for my experiments, mice continue to gain weight for up to about $1 \mathrm{yr}$ after birth. However, in mouse soleus there appear to be only very small differences in the relative proportions or absolute numbers of fast and slow myosin-containing fibers between mice of 1 week and 1 year even though large changes are obvious in rat soleus muscles over the same period (Wigston et al., 1989). Thus, unless there is a steady state in which equal numbers of fibers are converted from fast to slow as from slow to fast, remodeling of NMJs in mouse soleus is difficult to ascribe to a transformation of individual fibers from fast to slow fiber types since no such conversion appears to occur, at least after 1 week of age.

Another possibility that is consistent with the apparent difference in the extent of NMJ remodeling observed in soleus and LG is that NMJs in some muscles might undergo significant remodeling, whereas NMJs in other muscles might not. Since soleus contains a high proportion of slow-twitch or tonic muscle fibers, and LG is exclusively a fast-twitch or phasic fiber, it is conceivable that NMJs on slow-twitch fibers in soleus might exhibit more plasticity than NMJs in the fast-twitch LG muscle. In support of this hypothesis, little, if any, remodeling of NMJs has been observed in other exclusively fast-twitch muscles, for example, mouse sternomastoid (Lichtman et al., 1987; BaliceGordon and Lichtman, 1990) and bulbocavernosus (Breedlove et al., 1988), whereas significant NMJ remodeling has been reported for mouse pectineus, which contains large numbers of slow-twitch muscle fibers (Hill and Robbins, 1988). On the other hand, despite numerous controls and stringent conditions, it is still difficult to rule out the possibility that the synaptic remodeling observed is secondary to nerve or muscle damage. Indeed, 2 recent studies have shown that regions of presynaptic and postsynaptic sites can disappear if muscle fibers are transiently denervated and then reinnervated, or if muscle fibers degenerate and subsequently regenerate (Rich and Lichtman, 1989a, b).

An intriguing explanation for the apparent difference between fast- and slow-twitch muscles in the extent of NMJ remodeling is that remodeling might be activity-dependent. Changes in synaptic morphology have been observed in response to the im- 
position of altered patterns of neural activity (Pysh and Wiley, 1972; Lnenicka et al., 1986; Ficlds ct al., 1987) and following alterations in the use and effectiveness of a particular neural circuit (Bailey and Chen, 1983, 1988a, b, 1989). In addition, long-term changes in the efficacy of neuromuscular transmission following periods of nerve stimulation have been observed (Hinz and Wernig, 1988; Lnenicka and Atwood, 1988). The difference I have observed between the frequency of remodeling of NMJs in normal mouse soleus and LG might therefore reflect the more continuous activation of soleus NMJs compared with NMJs in fast-twitch LG (Hennig and Lomo, 1985). It should be possible to test this idea of activity-dependent synaptic remodeling at the mammalian neuromuscular junction, using in vivo imaging methods that cannot yet be feasibly applied to the less accessible synapses of the central nervous system.

\section{References}

Bailey CH, Chen M (1983) Morphological basis of long-term habituation and sensitization in Aplysia. Science 220:91-93.

Bailey CH, Chen M (1988a) Long-term memory in Aplysia modulates the total number of varicosities of single identified sensory neurons. Proc Natl Acad Sci USA 85:2373-2377.

Bailey CH, Chen M (1988b) Long-term sensitization in Aplysia increases the number of presynaptic contacts onto the identified gill motor neuron L7. Proc Natl Acad Sci USA 85:9356-9359.

Bailey $\mathrm{CH}$, Chen M (1989) Time course of structural changes at identified sensory neuron synapses during long-term sensitization in Aplysia. J Neurosci 9:1774-1780.

Balicc-Gordon RJ, Lichtman JW (1990) In vivo visualization of the growth of pre-and postsynaptic elements of neuromuscular junctions in the mouse. J Neurosci 10:894-908.

Breedlove SM, Balice-Gordon RJ, Lichtman JW (1988) Neuromuscular junctions shrink and expand as muscle fibers change size: studies in an androgen sensitive muscle. Soc Neurosci Abstr 14:1209.

Cardasis CA, Padykula HA (1981) Ultrastructural evidence indicating reorganization at the neuromuscular junction in the normal rat soleus muscle. Anat Rec 200:41-59.

Castellucci VF, Frost WN, Goelet P, Montarolo PG, Schacher S, Morgan JA, Blumenfield H, Kandel ER (1986) Cell and molecular analysis of long-term sensitization in Aplysia. J Physiol (Paris) 81:349-357.

Fahim MA, Holley JA, Robbins N (1984) Topographic comparison of neuromuscular junctions in slow and fast twitch muscles. Neuroscicnce 13:227-235.

Fields RD, Ellisman MH, Waxman SG (1987) Changes in synaptic morphology associated with presynaptic and postsynaptic activity: an in vitro study of the electrosensory organ of the Thomback ray. Synapse 1:335-346.

Greenough WT, Bailey GH (1988) The anatomy of a memory: convergence of results across a diversity of tests. Trends Neurosci 11 : 142-147.

Hennig R, Lomo T (1985) Firing patterns of motor units in normal rats. Nature 314:164-166.

Herrera AA, Banner LR, Nagaya N (1990) Repeated, in vivo observation of frog neuromuscular junctions: remodelling involves concurrent growth and retraction. J Neurocytol 19:85-99.

Hill R, Robbins N (1988) Dynamic structural rearrangements at the growing and adult mouse neuromuscular junction. Soc Neurosci Abstr 14:1208.
Hinz I, Wernig A (1988) Prolonged nerve stimulation causes changes in transmitter release at the frog neuromuscular junction. J Physiol 401:557-565.

Ito M (1989) Long-term depression: Annu Rev Neurosci 12:85-102.

Kugelberg E (1976) Adaptive transformation of rat soleus motor units during growth. J Neurol Sci 27:269-289.

Lichtman JW, Magrassi L, Purves D (1987) Visualization of neuromuscular junctions over periods of several months in living mice. $J$ Neurosci 7:1215-1222.

Lnenicka GA, Atwood HL (1988) Long-term changes in neuromuscular synapses with altered sensory input to a crayfish motoneuron. Exp Neurol 100:437-447.

Lnenicka GA, Atwood HL, Marin L (1986) Morphological transformation of synaptic terminals of a phasic motoneuron by long-term stimulation. J Neurosci 6:2252-2258.

Lomo T, Waerhaug O (1985) Motor endplates in fast and slow twitch muscles of the rat: what determines their differences? J Physiol (Paris) 80:290-297.

Magrassi L, Purves D, Lichtman JW (1987) Fluorescent probes that stain living nerve terminals. J Neurosci 7:1207-1214.

Nicoll RA, Kauer JA, Malenka RC (1988) The current excitement in long-term potentiation. Neuron 1:97-103.

Purves D, Voyvodic JT (1987) Imaging mammalian nerve cells and their connections over time in living animals. Trends Neurosci 10: 398-404.

Purves D, Voyvodic JT, Magrassi L, Yawo Y (1987) Nerve terminal remodeling visualized in living mice by repeated examination of the same neuron. Science 238:1122-1126.

Pysh JJ, Wiley RG (1972) Morphological alterations of synapses in electrically stimulated superior cervical ganglia of the cat. Science 176:191-193.

Rich MM, Lichtman JW (1989a) In vivo visualization of pre- and postsynaptic changes during synapse elimination in reinnervated mouse muscle. J Neurosci 9:1781-1805.

Rich MM, Lichtman JW (1989b) Motor nerve terminal loss from degenerating muscle fibers. Neuron 3:677-688.

Robbins N, Polak J (1987) Forms of growth and retraction at mouse neuromuscular junctions revealed by a new nerve terminal stain and correlative electron microscopy. Soc Neurosci Abstr 13:1007.

Rochel S, Robbins N (1987) Acetylcholine receptor availability and transmission efficiency. Brain Res 435:41-47.

Teyler TJ, DiScenna P (1987) Long-term potentiation. Annu Rev Neurosci 10:131-161.

Wernig A, Pecot-Déchavassine M, Stover H (1980) Sprouting and regression of the nerve at the frog neuromuscular junction in normal conditions and after prolonged paralysis with curare. J Neurocytol 9: 277-303.

Wernig A, Carmody JJ, Anzil AP, Hansert E, Marchiniak M, Zucker H (1984) Persistence of nerve sprouting with features of synapse remodelling in soleus muscles of adult mice. Neuroscience 11:241253.

Wigston DJ (1988) Direct visualization of synaptic remodeling in gastrocnemius muscles of living adult mice. Soc Neurosci Abstr 14: 1209.

Wigston DJ (1989) Remodeling of neuromuscular junctions in adult mouse soleus. J Neurosci 9:639-647.

Wigston DJ, Shirley KA, Schwartz G, English AW (1989) Fiber-type expression in rat and mouse soleus during postnatal development. Soc Neurosci Abstr 15:373.

Zucker RS (1989) Short-term synaptic plasticity. Annu Rev Neurosci 12:13-31. 